\title{
Three Methods for Revising Hybrid Knowledge Bases
}

\author{
Sebastian Binnewies, Zhiqiang Zhuang, and Kewen Wang \\ Griffith University, Institute for Integrated and Intelligent Systems, Australia \\ \{s.binnewies, z.zhuang, k. wang\}@griffith.edu.au
}

\begin{abstract}
Contemporary approaches for the Semantic Web include $h y$ brid knowledge bases that combine ontologies with rule-based languages. Despite a number of existing combination approaches, little attention has been given to change mechanisms for hybrid knowledge bases that can appropriately handle the dynamics of information on the Web. We present here three methods for revising hybrid knowledge bases in light of new information. We show by means of representation theorems that two of them fit properly into the classic belief change framework and that each of the two generalises the third method.
\end{abstract}

Keywords: Belief Revision, Hybrid Knowledge Bases, Logic Programs, Ontologies, Here-and-There Logic, Semantic Web

\section{Introduction}

In the last decade, ontologies [9] have become widely accepted as knowledge engineering artefacts in the Semantic Web for modelling domains of expertise. For example, SNOMED CT ${ }^{1}$, the Gene Ontology ${ }^{2}$, and OBO Foundry Ontologies ${ }^{3}$ represent standardised biomedical terminologies and are well-established within their field of application. They are usually specified in a fragment of first-order logic and can be viewed as first-order theories in restricted form. More recently, ontologies have been integrated with logic programs $[4,13]$ into hybrid knowledge bases to combine monotonic reasoning over the former component with nonmonotonic reasoning over the latter. A wide array of such integrations have been proposed (overviews can be found in $[7,12,14]$ ), which differ in the way ontologies and rules are combined. We concentrate here on hybrid knowledge bases under quantified here-and-there logic [3], because they place no restriction on the fragment of first-order logic or the class of logic programs to be used in the composition and capture several other integration approaches.

For hybrid knowledge bases to be successfully adopted in practical applications, it is crucial that they are not only static entities but also responsive to changes as required within the domain. It may be necessary to add new knowledge or retract some existing knowledge. We focus on this problem by framing

\footnotetext{
${ }^{1}$ http://www.snomed.org/snomed-ct

2 http://www.geneontology.org/

${ }^{3}$ http://www.obofoundry.org/
} 
it within the traditional belief change framework $[1,8,10]$, which provides sets of postulates that any rational change operator should satisfy. While the framework has been applied to a variety of knowledge representation formalisms (e.g., [2, 6, $23,24]$ ), a particular challenge in constructing appropriate belief change mechanisms for hybrid knowledge bases lies in their bi-component nature. Any change operator must be able to handle changes to the theory component, the logic program component, or both, depending on the new information acquired. When either component is changed, it may have an effect on the other component and cause the hybrid knowledge base to become inconsistent.

In the following, we address this gap by first adapting a partial meet revision operator from classical logics to hybrid knowledge bases. We then define a special case of partial meet revision, called protected revision, allowing to preferably revise only one component of the hybrid knowledge base. Finally, we present prioritised revision, a more fine-grained method, where a priority ordering over individual elements of the hybrid knowledge base determines the outcome. By evaluating our operators with respect to each other and the revision postulates, we find that partial meet revision and prioritised revision are interdefinable and comply with the belief change framework, whereas protected revision is a restricted version of partial meet revision.

\section{Preliminaries}

We first formally introduce hybrid knowledge bases under here-and-there logic and then briefly cover relevant belief change fundamentals.

\subsection{Hybrid Knowledge Bases under Here-and-There Logic}

Let $\mathcal{L}=\left(\mathcal{C}, \mathcal{P}_{T} \cup \mathcal{P}_{P}\right)$ be a function-free, first-order language, in which $\mathcal{C}$ is a set of constant symbols and $\mathcal{P}_{T}, \mathcal{P}_{P}$ are sets of predicate symbols such that $\mathcal{C}, \mathcal{P}_{T}, \mathcal{P}_{P}$ are pairwise disjoint. Let $\mathcal{L}$ further include the symbols ' $\wedge$ ', ' $\vee$ ', ' $\rightarrow$ ', ' $\neg$ ', ' $\forall$ ', ' $\exists$ ', the predicate symbol ' $\approx$ ' for equality, a countably infinite set of variables, and the standard punctuation marks, as well as the symbols ';', ', ', 'not', ' $\leftarrow$ ', and ' $\perp$ '. Atoms, formulas, closed formulas, and theories are defined in the usual way. A (first-order) rule $r$ over $\mathcal{L}$ has the form

$$
a_{1} ; \ldots ; a_{k} ; \text { not } b_{1} ; \ldots ; \text { not } b_{l} \leftarrow c_{1}, \ldots, c_{m}, \text { not } d_{1}, \ldots, \text { not } d_{n} .
$$

Here, all $a_{i}, b_{i}, c_{i}, d_{i}$ are atoms from $\mathcal{L}$ and $k, l, m, n \geq 0$. If $m=n=0$, then we omit ' $\leftarrow$ '. A hybrid knowledge base $(H K B)[3] K=\left(K_{T}, K_{P}\right)$ over $\mathcal{L}$ consists of a classical first-order theory $K_{T}$ over the language subset $\mathcal{L}_{T}=\left(\mathcal{C}, \mathcal{P}_{T}\right)$, and a set of rules of the form (1) over $\mathcal{L}$ that constitute a program $K_{P}$. We write $\mathcal{K}_{\mathcal{L}}$ for the class of all HKBs over $\mathcal{L}$.

A HKB can be used to combine specific constraints that hold in a domain, expressed by a program component $K_{P}$, with more general information, captured by a theory component $K_{T}$. For instance, a personal assistant system on a mobile phone may have in its HKB some general information about gender and marriage as well as some specific information about its user Chris, shown in Example 1. 
Example 1. Let $\mathcal{C}=\{$ Chris, Alex $\}, \mathcal{P}_{T}=\{$ male, female, married $\}, \mathcal{P}_{P}=\emptyset$, and $K=\left(K_{T}, K_{P}\right)$ be a HKB over $\left(\mathcal{C}, \mathcal{P}_{T} \cup \mathcal{P}_{P}\right)$ such that

$$
\begin{aligned}
K_{T}=\{ & \forall x: \text { male }(x) \rightarrow \neg \text { female }(x), \\
& \forall x, y: \text { married }(x, y) \rightarrow \text { married }(y, x), \\
& \forall x, y: \text { married }(x, y) \wedge \operatorname{female}(x) \rightarrow \operatorname{male}(y)\}, \\
K_{P}=\{ & \text { female }(\text { Chris }) .\} .
\end{aligned}
$$

We extend the usual set operations to HKBs as follows. For any pairs of sets $K^{1}=\left(K_{T}^{1}, K_{P}^{1}\right), \ldots, K^{n}=\left(K_{T}^{n}, K_{P}^{n}\right)$, let $K^{1} \odot \cdots \odot K^{n}=\left(\left\{K_{T}^{1} \odot \cdots \odot\right.\right.$ $\left.\left.K_{T}^{n}\right\},\left\{K_{P}^{1} \odot \cdots \odot K_{P}^{n}\right\}\right)$ for $\odot \in\{\cap, \cup\}, K^{1} \backslash K^{2}=\left(K_{T}^{1} \backslash K_{T}^{2}, K_{P}^{1} \backslash K_{P}^{2}\right)$, and for $i, j \in\{T, P\}$ with $i \neq j$ :

$$
\begin{gathered}
K^{1} \subseteq K^{2} \text { iff } K_{i}^{1} \subseteq K_{i}^{2} \text { and } K_{j}^{1} \subseteq K_{j}^{2}, \\
K^{1} \subseteq_{i} K^{2} \text { iff } K_{i}^{1} \subseteq K_{i}^{2} \text {, and if } K_{i}^{1}=K_{i}^{2} \text {, then } K_{j}^{1} \subseteq K_{j}^{2}, \text { and } \\
K^{1} \subset_{\circ} K^{2} \text { iff } K^{1} \subseteq_{\circ} K^{2} \text { and } K^{2} \nsubseteq_{\circ} K^{1} \text { where } \subseteq_{\circ} \in\left\{\subseteq, \subseteq_{T}, \subseteq_{P}\right\} .
\end{gathered}
$$

For any $K \in \mathcal{K}_{\mathcal{L}}$, let $\left.K\right|_{T}=\left(K_{T}, \emptyset\right),\left.K\right|_{P}=\left(\emptyset, K_{P}\right)$, and, for any $\mathbb{K} \subseteq 2^{K}$, $\left.\mathbb{K}\right|_{T}=\{(\tau, \emptyset) \mid(\tau, \rho) \in \mathbb{K}\}$ and $\left.\mathbb{K}\right|_{P}=\{(\emptyset, \rho) \mid(\tau, \rho) \in \mathbb{K}\}$.

An $\mathcal{L}$ structure is a pair $\mathcal{I}=(U, I)$, where the universe $U=(\mathcal{D}, \sigma)$ consists of a non-empty domain $\mathcal{D}$ and a function $\sigma$ from $\mathcal{C} \cup \mathcal{D}$ to $\mathcal{D}$ such that $\sigma(D)=D$ for all $D \in \mathcal{D}$, and an interpretation $I$ is a set of variable-free atoms that can be constructed from $\left(\mathcal{C} \cup \mathcal{D}, \mathcal{P}_{T} \cup \mathcal{P}_{P}\right)$. For a tuple $t=\left(D_{1}, \ldots, D_{n}\right)$, we define $\sigma(t)=\left(\sigma\left(D_{1}\right), \ldots, \sigma\left(D_{n}\right)\right)$. To obtain a ground instance of a rule in $K_{P}$, we replace each constant symbol $C$ in the rule by $\sigma(C)$ and each variable in the rule by an element from $\mathcal{D}$. The set of all ground instances of rules in $K_{P}$ is written $\operatorname{ground}\left(K_{P}\right)$.

A $\mathbf{Q H T}_{=}^{s}$ (quantified here-and-there logic with static domains and equality) structure with respect to $\mathcal{L}$ [18] (or simply $\mathbf{Q H T} \mathbf{T}_{=}^{s}$ structure if $\mathcal{L}$ is clear from the context) is a triple $\mathcal{M}=(U, H, T)$ such that $(U, H)$ and $(U, T)$ are $\mathcal{L}$ structures with $H \subseteq T$. Let $\mathcal{Q H T}$ be the set of all $\mathbf{Q H T}_{=}^{s}$ structures over $\mathcal{L}$. For any set $S$ of $\mathbf{Q H T}_{=}^{s}$ structures, by $\bar{S}$ we denote the complement of $S$ with respect to $\mathcal{Q H} \mathcal{T}$, that is, $\bar{S}=\mathcal{Q H} \mathcal{T} \backslash S$. We define the satisfaction relation for a $\mathbf{Q H T} \mathbf{T}_{=}^{s}$ structure $\mathcal{M}$ recursively as follows. Let $p\left(t_{1}, \ldots, t_{n}\right)$ be an atom and $\phi, \psi$ closed formulas built from $\left(\mathcal{C} \cup \mathcal{D}, \mathcal{P}_{T} \cup \mathcal{P}_{P}\right), x$ be a variable, and $w \in\{H, T\}$. Then

$$
\begin{aligned}
& \mathcal{M} \models_{w} p\left(t_{1}, \ldots, t_{n}\right) \text { iff } p\left(\sigma\left(t_{1}\right), \ldots, \sigma\left(t_{n}\right)\right) \in w, \\
& \mathcal{M} \models_{w} \phi \wedge \psi \text { iff } \mathcal{M}=_{w} \phi \text { and } \mathcal{M} \models_{w} \psi, \\
& \mathcal{M} \models_{w} \phi \vee \psi \text { iff } \mathcal{M}=_{w} \phi \text { or } \mathcal{M} \models_{w} \psi, \\
& \mathcal{M} \models_{T} \phi \rightarrow \psi \text { iff } \mathcal{M} \models_{T} \phi \text { or } \mathcal{M} \models_{T} \psi, \\
& \mathcal{M} \models_{H} \phi \rightarrow \psi \text { iff (i) } \mathcal{M} \models_{T} \phi \rightarrow \psi \text { and (ii) } \mathcal{M} \models_{H} \phi \text { or } \mathcal{M} \models_{H} \psi, \\
& \mathcal{M} \models_{w} \neg \phi \text { iff } \mathcal{M} \models_{T} \phi, \\
& \mathcal{M} \models_{T} \forall x \phi(x) \text { iff } \mathcal{M} \models_{T} \phi(D) \text { for all } D \in \mathcal{D}, \\
& \mathcal{M} \models_{H} \forall x \phi(x) \text { iff } \mathcal{M} \models_{T} \forall x \phi(x) \text { and } \mathcal{M} \models_{H} \phi(D) \text { for all } D \in \mathcal{D},
\end{aligned}
$$


$\mathcal{M} \models_{w} \exists x \phi(x)$ iff $\mathcal{M} \models_{w} \phi(D)$ for some $D \in \mathcal{D}$,

$\mathcal{M} \models_{w} t_{1} \approx t_{2}$ iff $\sigma\left(t_{1}\right)=\sigma\left(t_{2}\right)$.

A $\mathbf{Q H T} \mathbf{T}_{=}^{s}$ structure $\mathcal{M}$ is a $\mathbf{Q H T} \mathbf{T}_{=}^{s}$ model of a closed formula $\phi$, denoted $\mathcal{M} \models \phi$, iff $\overline{\mathcal{M}}==_{w} \phi$ for each $w \in\{H, T\}$. A $\mathbf{Q H T}_{=}^{s}$ structure $\mathcal{M}$ is a:

- $\mathbf{Q H T}_{=}^{s}$ model of a theory $K_{T}$ iff it is a model of all formulas in $K_{T}$;

- $\mathbf{Q H T}=$ model of a program $K_{P}$ iff it is a $\mathbf{Q H T}=$ model of all rules in $\operatorname{ground}\left(K_{P}\right)$ where each rule of the form (1) is interpreted as

$$
c_{1} \wedge \cdots \wedge c_{m} \wedge \neg d_{1} \wedge \cdots \wedge \neg d_{n} \rightarrow a_{1} \vee \cdots \vee a_{k} \vee \neg b_{1} \vee \cdots \vee \neg b_{l} ;
$$

- QHT $_{=}^{s}$ model of a HKB $K=\left(K_{T}, K_{P}\right)$ iff it is a $\mathbf{Q H T}_{=}^{s}$ model of $K_{T}$ and $K_{P}$.

The set of all $\mathbf{Q H T}_{=}^{s}$ models of $K$ is denoted by $Q H T(K)$ and $K$ is satisfiable iff $Q H T(K) \neq \emptyset$.

Example 2. For $K$ from Example 1, we obtain $Q H T(K)=\left\{\mathcal{M}_{1}, \mathcal{M}_{2}, \mathcal{M}_{3}, \mathcal{M}_{4}\right.$, $\left.\mathcal{M}_{5}, \mathcal{M}_{6}, \mathcal{M}_{7}, \mathcal{M}_{8}\right\}$ such that, for $i=\{1, \ldots, 8\}, U_{i}=(\{$ Chris, Alex $\}, \sigma)$ for each $\mathcal{M}_{i}$, where $\sigma$ is the identity function, and $H_{i}, T_{i}$ contain the atoms marked with a $H$ (here) or $T$ (there), respectively, in Table 1.

Table 1. $(H, T)$-Elements for Models of $K$ in Example 1

\begin{tabular}{|l|c|c|c|c|c|c|c|c|}
\hline & $\mathcal{M}_{1}$ & $\mathcal{M}_{2}$ & $\mathcal{M}_{3}$ & $\mathcal{M}_{4}$ & $\mathcal{M}_{5}$ & $\mathcal{M}_{6}$ & $\mathcal{M}_{7}$ & $\mathcal{M}_{8}$ \\
\hline male(Chris) & & & & & & & & \\
\hline male(Alex) & $\mathrm{H}, \mathrm{T}$ & $\mathrm{H}, \mathrm{T}$ & $\mathrm{T}$ & $\mathrm{H}, \mathrm{T}$ & $\mathrm{T}$ & & & \\
\hline female(Chris) & $\mathrm{H}, \mathrm{T}$ & $\mathrm{H}, \mathrm{T}$ & $\mathrm{H}, \mathrm{T}$ & $\mathrm{H}, \mathrm{T}$ & $\mathrm{H}, \mathrm{T}$ & $\mathrm{H}, \mathrm{T}$ & $\mathrm{H}, \mathrm{T}$ & $\mathrm{H}, \mathrm{T}$ \\
\hline female(Alex) & & & & & & $\mathrm{H}, \mathrm{T}$ & $\mathrm{T}$ & \\
\hline married(Chris, Alex) & $\mathrm{H}, \mathrm{T}$ & $\mathrm{T}$ & $\mathrm{T}$ & & & & & \\
\hline married(Alex,Chris) & $\mathrm{H}, \mathrm{T}$ & $\mathrm{T}$ & $\mathrm{T}$ & & & & & \\
\hline
\end{tabular}

\subsection{Belief Change}

The belief base framework $[8,10,19]$ of belief change defines expansion, revision, and contraction as the operations on a body of beliefs held by an agent, called a belief base, in light of some new information. In an expansion, new beliefs are simply added to an existing belief base, regardless of any inconsistencies that may arise. A revision operation also incorporates new beliefs into a belief base, but may discard some existing beliefs to ensure consistency of the outcome. During a contraction, some beliefs are removed from a belief base but no new beliefs are added. Particularly, and contrary to other frameworks, a belief base is 
not required to be closed under logical consequence, which makes this framework suitable for consideration regarding belief change in HKBs.

For propositional logic, Hansson [11] defined a partial meet (base) contraction operator $-_{\gamma}$ for a belief base $B$ and a sentence $\phi$ as $B-\gamma \phi=\bigcap \gamma(B \perp \phi)$, where $B \perp \phi=\left\{B^{\prime} \subseteq B \mid B^{\prime} \forall \phi\right.$ and, for all $B^{\prime \prime}, B^{\prime} \subset B^{\prime \prime} \subseteq B$ implies $\left.B^{\prime \prime} \vdash \phi\right\}$ and $\gamma$ is a selection function such that $\gamma(B \perp \phi)=\{B\}$ if $B \perp \phi=\emptyset$, and $\emptyset \neq \gamma(B \perp \phi) \subseteq B \perp \phi$ otherwise. He determined a set of four postulates that exactly characterises the class of partial meet contraction operators. He further defined a corresponding partial meet (base) revision operator $*_{\gamma}$ as $B *_{\gamma} \phi=$ $(B-\gamma \neg \phi) \cup\{\phi\}$ and showed that a set of five postulates exactly characterises the class of partial meet revision operators.

We now translate these five revision postulates to HKBs, so that we can later evaluate the rationality of our proposed operators. Let + be an expansion operator for $K \in \mathcal{K}_{\mathcal{L}}$ such that for any $K^{\prime} \in \mathcal{K}_{\mathcal{L}}, K+K^{\prime}=K \cup K^{\prime}$. The postulates are as follows, where we assume $K, K^{\prime}, K^{\prime \prime} \in \mathcal{K}_{\mathcal{L}}$ and a revision operator $*$ to be a function from $\mathcal{K}_{\mathcal{L}} \times \mathcal{K}_{\mathcal{L}}$ to $\mathcal{K}_{\mathcal{L}}$.

$(* 1) \quad K^{\prime} \subseteq K * K^{\prime}$

$(* 2) K * K^{\prime} \subseteq K+K^{\prime}$

(*3) If $\kappa \subseteq \bar{K} \backslash\left(K * K^{\prime}\right)$, then there exists a $\kappa^{\prime} \in \mathcal{K}_{\mathcal{L}}$ such that $K * K^{\prime} \subseteq \kappa^{\prime} \subset$ $K+K^{\prime}$ and $\kappa^{\prime}$ is satisfiable but $\kappa^{\prime} \cup \kappa$ is not satisfiable

$(* 4)$ If it holds for all $\kappa \subseteq K$ that $\kappa+K^{\prime}$ is satisfiable iff $\kappa+K^{\prime \prime}$ is satisfiable, then $K \cap\left(K * K^{\prime}\right)=K \cap\left(K * K^{\prime \prime}\right)$

$(* 5)$ If $K^{\prime}$ is satisfiable, then $K * K^{\prime}$ is satisfiable

\section{Revision in Hybrid Knowledge Bases}

In this section, we introduce three new methods for revising HKBs. We begin with an adaptation of partial meet revision (Section 3.1), then define a method that prioritises either component of a HKB in its entirety during a revision, called protected revision (Section 3.2), and finally propose a method that prioritises individual elements of a HKB, termed prioritised revision (Section 3.3).

\subsection{Partial Meet Revision}

As the foundation of our revision operations, we define the subsets of a HKB that are consistent with another HKB.

Definition 1 (Compatible Set). Let $K, K^{\prime} \in \mathcal{K}_{\mathcal{L}}$ and $\subseteq_{0} \in\left\{\subseteq, \subseteq_{T}, \subseteq_{P}\right\}$. The set of compatible sets of $K$ regarding $K^{\prime}$ is

$$
\begin{aligned}
& \mathbb{K}_{K^{\prime}, \subseteq_{\circ}}=\left\{\kappa=(\tau, \rho) \mid \kappa \subseteq K, Q H T(\kappa) \cap Q H T\left(K^{\prime}\right) \neq \emptyset\right. \text {, and } \\
& \text { for all } \kappa^{\prime}=\left(\tau^{\prime}, \rho^{\prime}\right), \kappa \subset_{0} \kappa^{\prime} \subseteq K \text { implies } \\
& \left.Q H T\left(\kappa^{\prime}\right) \cap Q H T\left(K^{\prime}\right)=\emptyset\right\} \text {. }
\end{aligned}
$$


To construct our revision, we will further make use of a selection function that chooses from a set of compatible sets the most suitable ones. As we aim to employ our selection function for different types of compatible sets, we define it here with respect to an arbitrary set.

Definition 2 (Selection Function). A selection function $\gamma$ for a set $S$ is a function such that i) $\mathbb{S} \subseteq 2^{S}$, ii) $\gamma(\mathbb{S}) \subseteq \mathbb{S}$, and iii) if $\mathbb{S} \neq \emptyset$, then $\gamma(\mathbb{S}) \neq \emptyset$.

We are now ready to introduce a partial meet revision operator for HKBs as follows.

Definition 3 (Partial Meet Revision). Let $K \in \mathcal{K}_{\mathcal{L}}$ and $\gamma$ be a selection function for $K$. A partial meet revision operator $*_{\gamma}$ for $K$ is defined such that for any $K^{\prime} \in \mathcal{K}_{\mathcal{L}}$ :

$$
K *_{\gamma} K^{\prime}= \begin{cases}K+K^{\prime} & \text { if } K^{\prime} \text { is not satisfiable, } \\ \bigcap \gamma\left(\mathbb{K}_{K^{\prime}, \subseteq}\right)+K^{\prime} & \text { otherwise. }\end{cases}
$$

Since one of the maxims for belief revision is to ensure primacy of new information [5], our revision operator $*_{\gamma}$ always includes the information expressed by the revising HKB $K^{\prime}$ in the outcome. In addition, the main characteristic that distinguishes a revision from an expansion is that the former aims at providing a consistent outcome. In the case that $K^{\prime}$ is itself not satisfiable, our revision operator returns the combination of $K^{\prime}$ and the initial HKB $K$ in its entirety, since no removal of any part of $K$ would lead to a satisfiable outcome. This behaviour adheres to the maxim of persistence of prior knowledge [5]. In all other cases, the revision operator returns the intersection of the selected compatible sets of $K$ added to $K^{\prime}$.

We obtain the following representation theorem, stating that our partial meet revision operator $*_{\gamma}$ is exactly characterised by the postulates $(* 1)-(* 5)$.

Lemma 1. Let $K, K^{\prime} \in \mathcal{K}_{\mathcal{L}}$ and $\gamma$ be a selection function for $K$. If $\kappa \subseteq K \cap K^{\prime}$, then $\kappa \subseteq$ ○ी $\gamma\left(\mathbb{K}_{K^{\prime}, \subseteq_{\circ}}\right)$.

Theorem 1. An operator $*_{\gamma}$ is a partial meet revision operator for $K \in \mathcal{K}_{\mathcal{L}}$ determined by a selection function $\gamma$ for $K$ iff $*_{\gamma}$ satisfies $(* 1)-(* 5)$.

Proof. We first show that a partial meet revision operator $*_{\gamma}$ for $K$ determined by a given selection function $\gamma$ for $K$ satisfies $(* 1)-(* 5)$.

$(* 1)$ : Follows directly from Definition 3 .

$(* 2)$ : If $K^{\prime}$ is not satisfiable, then $K *_{\gamma} K^{\prime}=K+K$ by Definition 3. Otherwise, since $\bigcap \gamma\left(\mathbb{K}_{K^{\prime}, \subseteq}\right) \subseteq K$ we have $\bigcap \gamma\left(\mathbb{K}_{K^{\prime}, \subseteq}\right)+K^{\prime} \subseteq K+K^{\prime}$.

$(* 3)$ : Let $\kappa \subseteq K$. Assume that for all $\kappa^{\prime}$ with $K *_{\gamma} K^{\prime} \subseteq \kappa^{\prime} \subset K+K^{\prime}$ and $\kappa^{\prime}$ being satisfiable, it holds that $\kappa^{\prime} \cup \kappa$ is satisfiable. In particular, for each $\kappa^{\prime \prime} \in \mathbb{K}_{K^{\prime}, \subseteq}$ with $K *_{\gamma} K^{\prime} \subseteq \kappa^{\prime \prime} \cup K^{\prime}$, this implies $\kappa^{\prime \prime} \cup K^{\prime} \cup \kappa$ is satisfiable. As each $\kappa^{\prime \prime}$ is $\subseteq$-maximal, it follows that $\kappa \subseteq \kappa^{\prime \prime}$ and thus $\kappa \subseteq \bigcap \gamma\left(\mathbb{K}_{K^{\prime}, \subseteq}\right)$. From Definition 3 we can then conclude $\kappa \nsubseteq K \backslash\left(K *_{\gamma} K^{\prime}\right)$. 
$(* 4)$ : For all $\kappa \subseteq K$, let $\kappa+K^{\prime}$ be satisfiable iff $\kappa+K^{\prime \prime}$ is satisfiable. Then $\mathbb{K}_{K^{\prime}, \subseteq}=\mathbb{K}_{K^{\prime \prime}, \subseteq}$ by Definition 1 and so $\bigcap \gamma\left(\mathbb{K}_{K^{\prime}, \subseteq}\right)=\bigcap \gamma\left(\mathbb{K}_{K^{\prime \prime}, \subseteq}\right)$ as well as $K \cap \bigcap \gamma\left(\mathbb{K}_{K^{\prime}, \subseteq}\right)=K \cap \bigcap \gamma\left(\mathbb{K}_{K^{\prime \prime}, \subseteq}\right)$. By Lemma 1 we obtain $\left(K \cap \bigcap \gamma\left(\mathbb{K}_{K^{\prime}, \subseteq}\right)\right) \cup$ $\left(K \cap K^{\prime}\right)=\left(K \cap \bigcap \gamma\left(\mathbb{K}_{K^{\prime \prime}, \subseteq}\right)\right) \cup\left(K \cap K^{\prime \prime}\right)$. This means $K \cap\left(\bigcap \gamma\left(\mathbb{K}_{K^{\prime}, \subseteq}\right) \cup K^{\prime}\right)=$ $K \cap\left(\bigcap \gamma\left(\mathbb{K}_{K^{\prime \prime}, \subseteq}\right) \cup K^{\prime \prime}\right)$. Thus, $K \cap\left(K *_{\gamma} K^{\prime}\right)=K \cap\left(K *_{\gamma} K^{\prime \prime}\right)$.

$(* 5)$ : If $K^{\prime}$ is satisfiable, then, for any $\kappa \in \mathbb{K}_{K^{\prime}, \subseteq}, \kappa+K^{\prime}$ is satisfiable, which implies $K *_{\gamma} K^{\prime}$ is satisfiable.

We now show that any operator $\circ_{\gamma}$ for $K$ satisfying $(* 1)-(* 5)$ is a partial meet revision operator for $K$ determined by some selection function for $K$. We first find a selection function $\gamma$ for $K$. Let $\gamma$ be such that (i) if $\mathbb{K}_{K^{\prime}, \subseteq}=\emptyset$, then $\gamma\left(\mathbb{K}_{K^{\prime}, \subseteq}\right)=\emptyset$ and (ii) $\gamma\left(\mathbb{K}_{K^{\prime}, \subseteq}\right)=\left\{\kappa \in \mathbb{K}_{K^{\prime}, \subseteq} \mid K \cap\left(K \circ_{\gamma} K^{\prime}\right) \subseteq \kappa\right\}$ otherwise.

We begin by showing that $\gamma$ is a function. For any $K^{\prime}, K^{\prime \prime} \in \mathcal{K}_{\mathcal{L}}$, if $\mathbb{K}_{K^{\prime}, \subseteq}=$ $\mathbb{K}_{K^{\prime \prime}, \subseteq}$, then $K \cap\left(K \circ_{\gamma} K^{\prime}\right)=K \cap\left(K \circ_{\gamma} K^{\prime \prime}\right)$ by $(* 4)$. This means $\gamma\left(\mathbb{K}_{K^{\prime}, \subseteq}\right)=$ $\gamma\left(\mathbb{K}_{K^{\prime \prime}, \subseteq}\right)$ according to our definition of $\gamma$.

We next show that $\gamma$ is a selection function. Clearly, $\gamma\left(\mathbb{K}_{\left.K^{\prime}, \subset\right)} \subseteq \mathbb{K}_{K^{\prime}, \subset}\right.$ by

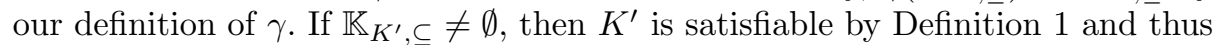
$K \circ_{\gamma} K^{\prime}$ is satisfiable by $(* 5)$. Since $K^{\prime} \subseteq K \circ_{\gamma} K^{\prime}$ by $(* 1)$ and $K \circ_{\gamma} K^{\prime} \subseteq K \cup K^{\prime}$ by $(* 2)$, it follows that $\left(K \cap\left(K \circ_{\gamma} K^{\prime}\right)\right) \cup K^{\prime}$ is satisfiable. This means that there

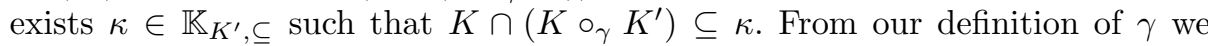
therefore obtain that $\gamma\left(\mathbb{K}_{K^{\prime}, \subseteq}\right) \neq \emptyset$.

Finally, we show that $o_{\gamma}$ is a partial meet revision operator for $K$, that is, $K \circ_{\gamma} K^{\prime}=K \cup K^{\prime}$ if $K^{\prime}$ is not satisfiable and $K \circ_{\gamma} K^{\prime}=\bigcap \gamma\left(\mathbb{K}_{K^{\prime}, \subseteq}\right) \cup K^{\prime}$ otherwise. Consider first the limiting case that $K^{\prime}$ is not satisfiable. If $\kappa \subseteq K \backslash\left(K \circ \circ_{\gamma} K^{\prime}\right)$, then there exists $\kappa^{\prime}$ such that $K \circ_{\gamma} K^{\prime} \subseteq \kappa^{\prime} \subset K \cup K^{\prime}$ and $\kappa^{\prime}$ is satisfiable but $\kappa^{\prime} \cup \kappa$ is not satisfiable by $(* 3)$. This is a contradiction since $K^{\prime} \subseteq \kappa^{\prime}$ by $(* 1)$. Therefore, it holds for all $\kappa \subseteq K$ that $\kappa \subseteq K \circ_{\gamma} K^{\prime}$, that is, $K \subseteq K \circ_{\gamma} K^{\prime}$. Since $K^{\prime} \subseteq K \circ_{\gamma} K^{\prime}$ by $(* 1)$ and $K \circ_{\gamma} K^{\prime} \subseteq K \cup K^{\prime}$ by $(* 2)$, we can conclude $K \circ_{\gamma} K^{\prime}=K \cup K^{\prime}$.

Assume now that $K^{\prime}$ is satisfiable. Let $\kappa \subseteq K \backslash\left(K \circ_{\gamma} K^{\prime}\right)$. If $\mathbb{K}_{K^{\prime}, \subseteq}=\emptyset$, then it follows from $(* 1)$ and $(* 3)$ that $K \circ_{\gamma} K^{\prime}=K^{\prime}$. Since $\gamma\left(\mathbb{K}_{K^{\prime}, \subseteq}\right) \stackrel{=}{=}$ by our definition of $\gamma$, we thus have $K \circ_{\gamma} K^{\prime}=K^{\prime}=\bigcap \gamma\left(\mathbb{K}_{K^{\prime}, \subseteq}\right) \cup K^{\prime}$. If $\mathbb{K}_{K^{\prime}, \subseteq \neq \emptyset \text {, then }}$ it follows directly from our definition of $\gamma$ that $K \cap\left(K \circ_{\gamma} K^{\prime}\right) \subseteq \bigcap \gamma\left(\mathbb{K}_{K^{\prime}, \subseteq}\right)$. From $(* 1)$ and $(* 2)$ we then obtain $K \circ_{\gamma} K^{\prime} \subseteq \bigcap \gamma\left(\mathbb{K}_{K^{\prime}, \subseteq}\right) \cup K^{\prime}$. To show the converse inclusion, first assume the case that $K \cup K^{\prime}$ is satisfiable. This implies that for any $\kappa^{\prime} \subseteq K \cup K^{\prime}$ it holds that $\kappa^{\prime}$ is satisfiable. Applying $(* 3)$, we obtain $K \backslash\left(K \circ_{\gamma} K^{\prime}\right)=$ $\emptyset$ and thus $K \subseteq K \circ_{\gamma} K^{\prime}$. From $(* 1)$ and $(* 2)$ it follows that $K \circ_{\gamma} K^{\prime}=K \cup K^{\prime}$. Moreover, due to the assumption that $K \cup K^{\prime}$ is satisfiable and Definition 1, we have $\mathbb{K}_{K^{\prime}, \subseteq}=\{K\}$. By our definition of $\gamma$, we obtain $\gamma\left(\mathbb{K}_{K^{\prime}, \subseteq}\right)=\{K\}$ and thus $\bigcap \gamma\left(\mathbb{K}_{K^{\prime}, \subseteq}\right)=K$ and can conclude $K \circ_{\gamma} K^{\prime}=\bigcap \gamma\left(\mathbb{K}_{K^{\prime}, \subseteq}\right) \cup K^{\prime}$. Lastly, assume the case that $K \cup K^{\prime}$ is not satisfiable. We will show that $\kappa \nsubseteq K \circ_{\gamma} K^{\prime}$ implies $\kappa \nsubseteq \bigcap \gamma\left(\mathbb{K}_{K^{\prime}, \subseteq}\right) \cup K^{\prime}$. If $\kappa \nsubseteq K$, then $\kappa \nsubseteq\left(K \circ_{\gamma} K^{\prime}\right) \backslash K^{\prime}$ by $(* 1)$ and $(* 2)$ and $\kappa \nsubseteq \bigcap \gamma\left(\mathbb{K}_{\left.K^{\prime}, \subseteq\right)}\right)$ by Definition 1. Since $\kappa \nsubseteq K \circ_{\gamma} K^{\prime}$ implies $\kappa \nsubseteq K^{\prime}$ by $(* 1)$, it follows that $\left.\kappa \nsubseteq\left(\left(K \circ_{\gamma} K^{\prime}\right) \backslash K^{\prime}\right) \cup K^{\prime}\right)=K \circ_{\gamma} K^{\prime}$ and $\kappa \subseteq \bigcap \gamma\left(\mathbb{K}_{K^{\prime}, \subseteq}\right) \cup K^{\prime}$. Now assume $\kappa \subseteq K \backslash\left(K \circ_{\gamma} K^{\prime}\right)$. According to $(* 3)$, then there exists $\kappa^{\prime}$ such that $K \circ_{\gamma} K^{\prime} \subseteq \kappa^{\prime} \subset K \cup K^{\prime}$ and $\kappa^{\prime}$ is satisfiable but $\kappa^{\prime} \cup \kappa$ is not satisfiable. 


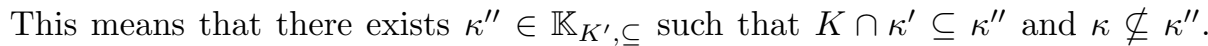
Since $K \cap\left(K \circ \circ_{\gamma} K^{\prime}\right) \subseteq K \cap \kappa^{\prime} \subseteq \kappa^{\prime \prime}$, we obtain from our definition of $\gamma$ that $\kappa^{\prime \prime} \in \gamma\left(\mathbb{K}_{K^{\prime}, \subseteq}\right)$. We can thus conclude from $\kappa \nsubseteq \kappa^{\prime \prime}$ that $\kappa \nsubseteq \bigcap \gamma\left(\mathbb{K}_{K^{\prime}, \subseteq}\right)$.

\subsection{Protected Revision}

As a HKB consists of two components, it may be desirable to emphasise one component over the other during a revision operation. We can achieve this by a protected revision, which keeps the theory (or program) component of the initial HKB intact as much as possible whenever the theory (or program) component of the revising HKB is compatible with the initial HKB.

Definition 4 (Protected Revision). Let $K \in \mathcal{K}_{\mathcal{L}}$ and $\gamma$ be a selection function for $K$. A protected revision operator $*_{\gamma, \subseteq_{0}}$ for $K$ is defined such that for any $K^{\prime} \in \mathcal{K}_{\mathcal{L}}$ :

$$
K *_{\gamma, \subseteq_{0}} K^{\prime}= \begin{cases}K+K^{\prime} & \text { if } K^{\prime} \text { is not satisfiable, } \\ \bigcap \gamma\left(\mathbb{K}_{K^{\prime}, \subseteq_{0}}\right)+K & \text { otherwise, }\end{cases}
$$

where $\subseteq_{0}=\subseteq_{T}$ if $K+\left.K^{\prime}\right|_{T}$ is satisfiable, $\subseteq_{0}=\subseteq_{P}$ if $K+\left.K^{\prime}\right|_{P}$ is satisfiable, and $\subseteq_{0}=\subseteq$ otherwise.

In contrast to our partial meet revision operator, the protected revision operator $*_{\gamma}, \subseteq_{\circ}$ relies on selecting only among those compatible sets that have a maximal theory (or program) component, whenever $K+\left.K^{\prime}\right|_{T}\left(K+\left.K^{\prime}\right|_{P}\right.$, respectively) is satisfiable. The two revision operators coincide whenever neither $K+\left.K^{\prime}\right|_{T}$ nor $K+\left.K^{\prime}\right|_{P}$ is satisfiable. Obviously, the parameter $\subseteq_{\circ}$ could also be fixed to $\subseteq_{T}$ (or $\subseteq_{P}$ ) in settings where the theory (program, respectively) component is required to have absolute priority over the other. We illustrate the operation of protected revision in the following example.

Example 3. Consider again $K$ from Example 1. Suppose the user Chris now states the command: "Call my wife Alex." This input by the user contains new information, i.e., Alex is female and Chris is married to Alex, to be added to $K$. Formally, let $K^{\prime}=\left(K_{T}^{\prime}, K_{P}^{\prime}\right) \in \mathcal{K}_{\mathcal{L}}$ with $K_{T}^{\prime}=\emptyset$ and $K_{P}^{\prime}=\{$ female $($ Alex $)$., married (Chris, Alex). $\}$. Since $K_{T}^{\prime}=\emptyset, K+\left.K^{\prime}\right|_{T}$ is satisfiable and we obtain $\mathbb{K}_{K^{\prime}, \subseteq_{T}}=\left\{\left(K_{T}, \emptyset\right)\right\}$. Thus, $K *_{\gamma, \subseteq_{T}} K^{\prime}=\left(K_{T}, K_{P}^{\prime}\right)$.

The next proposition establishes that partial meet revision is a generalisation of protected revision.

Proposition 1. Let $K, K^{\prime} \in \mathcal{K}_{\mathcal{L}}$. For any selection function $\gamma$ for $K$, there exists a selection function $\gamma^{\prime}$ for $K$ such that $K *_{\gamma, \subseteq_{0}} K^{\prime}=K *_{\gamma^{\prime}} K^{\prime}$.

Proof. Let $K, K^{\prime} \in \mathcal{K}_{\mathcal{L}}$ and $\gamma$ be a selection function for $K$. In the limiting case that $K^{\prime}$ is not satisfiable, it holds by Definitions 3 and 4 that $K *_{\gamma, \subseteq_{0}} K^{\prime}=$ $K+K^{\prime}=K *_{\gamma} K^{\prime}$ for any selection function $\gamma^{\prime}$. Otherwise, since $\mathbb{K}_{K^{\prime}, \subseteq} \subseteq \mathbb{K}_{K^{\prime}, \subseteq}$ for $\subseteq_{\circ} \in\left\{\subseteq, \subseteq_{T}, \subseteq_{P}\right\}$ by Definition 1 , we can choose a selection function $\gamma^{\prime}$ for $K$ such that $\gamma^{\prime}\left(\mathbb{K}_{K^{\prime}, \subseteq}\right)=\gamma\left(\mathbb{K}_{K^{\prime}, \subseteq_{0}}\right)$. From Definitions 3 and 4 it then follows that $K *_{\gamma, \subseteq_{\circ}} K^{\prime}=K *_{\gamma^{\prime}} K^{\prime}$. 
The other direction of the proposition does not hold because the use of the parameter $\subseteq_{\circ}$ in Definition 4 restricts the compatible sets to be included in $\mathbb{K}_{K^{\prime}, \subseteq_{0}}$. Due to this restriction, protected revision also does not satisfy $(* 4)$. If we consider the weaker postulate $(* 4 \mathrm{c})$ below, then we can show that our protected revision operator $*_{\gamma}, \subseteq_{\circ}$ satisfies $(* 1)-(* 3),(* 4 \mathrm{c})$, and $(* 5)$.

(*4c) If it holds for all $\kappa \subseteq K$ that $\kappa+\left.K^{\prime}\right|_{T}$ is satisfiable iff $\kappa+\left.K^{\prime \prime}\right|_{T}$ is satisfiable and $\kappa+\left.K^{\prime}\right|_{P}$ is satisfiable iff $\kappa+\left.K^{\prime \prime}\right|_{P}$ is satisfiable, then $K \cap\left(K * K^{\prime}\right)=$ $K \cap\left(K * K^{\prime \prime}\right)$

Theorem 2. Let $K \in \mathcal{K}_{\mathcal{L}}$ and $\gamma$ be a selection function for $K$. If $*_{\gamma, \subseteq_{\circ}}$ is a protected revision operator for $K$, then $*_{\gamma}, \subseteq_{0}$ satisfies $(* 1)-(* 3),(* 4 c)$, and $(* 5)$.

Proof. $(* 1)-(* 3)$ and $(* 5)$ : Follow analogously to the proofs for Theorem 1 .

$(* 4 \mathrm{c})$ : Assume that for all $\kappa \subseteq K$, it holds that $\kappa+\left.K^{\prime}\right|_{T}$ is satisfiable iff $\kappa+\left.K^{\prime \prime}\right|_{T}$ is satisfiable and $\kappa+\left.K^{\prime}\right|_{P}$ is satisfiable iff $\kappa+\left.K^{\prime \prime}\right|_{P}$ is satisfiable. We proceed by cases.

Case 1: $K+\left.K^{\prime}\right|_{T}$ is satisfiable. This implies $K+\left.K^{\prime \prime}\right|_{T}$ is satisfiable by the assumption and thus $\mathbb{K}_{\left.K^{\prime}\right|_{T}, \subset_{0}}=K=\mathbb{K}_{\left.K^{\prime \prime}\right|_{T}, \subseteq_{0}}$ by Definition 1. In particular, $\mathbb{K}_{\left.K^{\prime}\right|_{T}, \subseteq_{T}}=K=\mathbb{K}_{\left.K^{\prime \prime}\right|_{T}, \subseteq_{T}}$ as Definition 4 requires $\subseteq_{0}=\subseteq_{T}$. Since $\kappa+\left.K^{\prime}\right|_{P}$ is satisfiable iff $\kappa+\left.K^{\prime \prime}\right|_{P}$ is satisfiable for all $\kappa \subseteq K$ by the assumption, we obtain $\mathbb{K}_{K^{\prime}, \subseteq_{T}}=\mathbb{K}_{K^{\prime \prime}, \subseteq_{T}}$ by Definition 1. Then $\bigcap \gamma\left(\mathbb{K}_{K^{\prime}, \subseteq_{T}}\right)=\bigcap \gamma\left(\mathbb{K}_{K^{\prime \prime}, \subseteq_{T}}\right)$ as well as $K \cap \bigcap \gamma\left(\mathbb{K}_{K^{\prime}, \subseteq_{T}}\right)=K \cap \bigcap \gamma\left(\mathbb{K}_{K^{\prime \prime}, \subseteq_{T}}\right)$. By Lemma 1 we obtain $(K \cap$ $\left.\bigcap \gamma\left(\mathbb{K}_{K^{\prime}, \subseteq_{T}}\right)\right) \cup\left(K \cap K^{\prime}\right)=\left(K \cap \bigcap \gamma\left(\mathbb{K}_{K^{\prime \prime}, \subseteq_{T}}\right)\right) \cup\left(K \cap K^{\prime \prime}\right)$. This means $K \cap$ $\left(\bigcap \gamma\left(\mathbb{K}_{K^{\prime}, \subseteq_{T}}\right) \cup K^{\prime}\right)=K \cap\left(\bigcap \gamma\left(\mathbb{K}_{K^{\prime \prime}, \subseteq_{T}}\right) \cup K^{\prime \prime}\right)$. Thus, $K \cap\left(K *_{\gamma, \subseteq_{T}} K^{\prime}\right)=$ $K \cap\left(K *_{\gamma, \subseteq_{T}} K^{\prime \prime}\right)$.

Case 2: $K+\left.K^{\prime}\right|_{P}$ is satisfiable. Follows symmetrically to Case 1.

Case 3: Neither $K+\left.K^{\prime}\right|_{T}$ nor $K+\left.K^{\prime}\right|_{P}$ is satisfiable. This implies neither $K+\left.K^{\prime \prime}\right|_{T}$ nor $K+\left.K^{\prime \prime}\right|_{P}$ is satisfiable by the assumption as well as $\subseteq_{\circ}=\subseteq$ by Definition 4 . Thus, $*_{\gamma}, \subseteq_{0}=*_{\gamma}$ and the proof reduces to the one for satisfaction of $(* 4)$ in Theorem 1 .

At this point it becomes interesting to investigate how protected revision relates to a "stepwise" application of partial meet revision, that is, $\left.K\right|_{P} *_{\gamma}\left(\left.K\right|_{T} *_{\gamma}\right.$ $\left.K^{\prime}\right)$ or $\left.K\right|_{T *_{\gamma}}\left(\left.K\right|_{P} *_{\gamma} K^{\prime}\right)$. It turns out that the result of protected revision $K *_{\gamma}, \subseteq_{T} K^{\prime}$ is a subset of the result of stepwise revision $\left.K\right|_{P} *_{\gamma}\left(\left.K\right|_{T} *_{\gamma} K^{\prime}\right)$, provided that the selection function chooses similar sets in each respective operation, more specifically, the same theory-maximal compatible sets for protected revision as for the first step of stepwise revision and only those compatible sets for the second step of stepwise revision that include the program component of a selected compatible set for protected revision (analogously for $\left.K\right|_{T^{*}}\left(\left.K\right|_{P^{*}{ }_{\gamma}} K^{\prime}\right.$ ) and $\left.K *_{\gamma, \subseteq_{P}} K^{\prime}\right)$. Before we show these results formally in Propositions 2 and 3 , we introduce some useful notation. For any $K, K^{\prime} \in \mathcal{K}_{\mathcal{L}}$, let

$$
\begin{aligned}
& \mathbb{T}_{K^{\prime}}=\{(\tau, \emptyset) \mid \subseteq K_{T}, Q H T((\tau, \emptyset)) \cap Q H T\left(K^{\prime}\right) \neq \emptyset, \text { and, for all } \tau^{\prime}, \\
& \tau\left.\subset \tau^{\prime} \subseteq K_{T} \text { implies } Q H T\left(\left(\tau^{\prime}, \emptyset\right)\right) \cap Q H T\left(K^{\prime}\right)=\emptyset\right\} \\
& \mathbb{P}_{K^{\prime}}=\left\{(\emptyset, \rho) \mid \rho \subseteq K_{P}, Q H T((\emptyset, \rho)) \cap Q H T\left(K^{\prime}\right) \neq \emptyset, \text { and, for all } \rho^{\prime},\right.
\end{aligned}
$$




$$
\left.\rho \subset \rho^{\prime} \subseteq K_{P} \text { implies } Q H T\left(\left(\emptyset, \rho^{\prime}\right)\right) \cap Q H T\left(K^{\prime}\right)=\emptyset\right\} .
$$

Proposition 2. Let $K, K^{\prime} \in \mathcal{K}_{\mathcal{L}}$ and $K+\left.K^{\prime}\right|_{T}$ be satisfiable. There exists a selection function $\gamma$ for $K$ such that $\left.K *_{\gamma, \subseteq_{T}} K^{\prime} \subseteq K\right|_{P} *_{\gamma}\left(\left.K\right|_{T} *_{\gamma} K^{\prime}\right)$.

Proof. Let $K, K^{\prime} \in \mathcal{K}_{\mathcal{L}}$ and $K+\left.K^{\prime}\right|_{T}$ be satisfiable. Assume that $\gamma$ is a selection function for $K$ such that $\gamma\left(\mathbb{T}_{K^{\prime}}\right)=\left.\gamma\left(\mathbb{K}_{K^{\prime}, \subset_{T}}\right)\right|_{T}$ and $\gamma\left(\mathbb{P}_{\left.K\right|_{T} *_{\gamma} K^{\prime}}\right)=\{(\emptyset, \rho) \mid$ $\left.\left(\emptyset, \rho^{\prime}\right) \in \gamma\left(\mathbb{K}_{K^{\prime}, \subseteq_{T}}\right)\right|_{P}$ and $\left.\left(\emptyset, \rho^{\prime}\right) \subseteq(\emptyset, \rho)\right\}$. If $K^{\prime}$ is not satisfiable, then it follows directly from Definitions 3 and 4 that $K *_{\gamma}, \subseteq_{T} K^{\prime}=K+K^{\prime}=\left.K\right|_{P} *_{\gamma}\left(\left.K\right|_{T} *_{\gamma} K^{\prime}\right)$. Otherwise, it follows from Definition 1 and our definition of $\mathbb{T}_{K^{\prime}}$ that $\mathbb{T}_{K^{\prime}}=$ $\left.\mathbb{K}_{K^{\prime}, \subseteq_{T}}\right|_{T}$. Due to the assumption, this implies $\bigcap \gamma\left(\mathbb{T}_{K^{\prime}}\right)=\left.\bigcap \gamma\left(\mathbb{K}_{K^{\prime}, \subseteq_{T}}\right)\right|_{T}$. For each $\left.(\tau, \emptyset) \in \gamma\left(\mathbb{K}_{K^{\prime}, \subseteq_{T}}\right)\right|_{T}$, since $\bigcap \gamma\left(\mathbb{T}_{K^{\prime}}\right) \subseteq(\tau, \emptyset)$ it holds that $Q H \bar{T}((\tau, \emptyset)) \cap$ $Q H T\left(K^{\prime}\right) \subseteq Q H T\left(\bigcap \gamma\left(\mathbb{T}_{K^{\prime}}\right)\right) \cap Q H T\left(K^{\prime}\right)$. Thus, for each $\left.(\emptyset, \rho) \in \gamma\left(\mathbb{K}_{K^{\prime}, \subseteq_{T}}\right)\right|_{P}$ there exists a $\left(\emptyset, \rho^{\prime}\right) \in \mathbb{P}_{\left.K\right|_{T^{*}} K^{\prime}}$ such that $(\emptyset, \rho) \subseteq\left(\emptyset, \rho^{\prime}\right)$. From the assumption we then obtain $\left.\bigcap \gamma\left(\mathbb{K}_{K^{\prime}, \subseteq_{T}}\right)\right|_{P} \subseteq \bigcap \gamma\left(\mathbb{P}_{\left.K\right|_{T} *_{\gamma} K^{\prime}}\right)$, which implies $K *_{\gamma, \subseteq_{T}} K^{\prime} \subseteq$ $\left.K\right|_{P} *_{\gamma}\left(\left.K\right|_{T} *_{\gamma} K^{\prime}\right)$ by Definitions 3 and 4 .

Proposition 3. Let $K, K^{\prime} \in \mathcal{K}_{\mathcal{L}}$ and $K+\left.K^{\prime}\right|_{P}$ be satisfiable. There exists a selection function $\gamma$ for $K$ such that $\left.K *_{\gamma, \subseteq_{P}} K^{\prime} \subseteq K\right|_{T} *_{\gamma}\left(\left.K\right|_{P} *_{\gamma} K^{\prime}\right)$.

Proof. Let $K, K^{\prime} \in \mathcal{K}_{\mathcal{L}}$ and $K+\left.K^{\prime}\right|_{P}$ be satisfiable. Assume that $\gamma$ is a selection function for $K$ such that $\gamma\left(\mathbb{P}_{K^{\prime}}\right)=\left.\gamma\left(\mathbb{K}_{K^{\prime}, \subseteq_{P}}\right)\right|_{P}$ and $\gamma\left(\mathbb{T}_{\left.K\right|_{P} *_{\gamma} K^{\prime}}\right)=\{(\tau, \emptyset) \mid$ $\left.\left(\tau^{\prime}, \emptyset\right) \in \gamma\left(\mathbb{K}_{K^{\prime}, \subseteq_{P}}\right)\right|_{T}$ and $\left.\left(\tau^{\prime}, \emptyset\right) \subseteq(\tau, \emptyset)\right\}$. The proof then follows symmetrically to the proof of Proposition 2.

For completeness, we also show here the relationship between stepwise revision and partial meet revision in Propositions 4 and 5, namely, that the result of stepwise revision is a subset of the result of partial meet revision, given that the selection function behaves similarly in each operation. Particularly, this is the case for $\left.K\right|_{P} *_{\gamma}\left(\left.K\right|_{T} *_{\gamma} K^{\prime}\right)$ whenever the theory part of $K$ retained in the first step of stepwise revision is included in each selected compatible set for partial meet revision and the set of selected compatible sets for the second step of stepwise revision is the same as the set of program parts in the selected compatible sets for partial meet revision (analogously for $\left.K\right|_{T} *_{\gamma}\left(\left.K\right|_{P} *_{\gamma} K^{\prime}\right)$ ).

Proposition 4. Let $K, K^{\prime} \in \mathcal{K}_{\mathcal{L}}$. There exists a selection function $\gamma$ for $K$ such that $\left.K\right|_{P} *_{\gamma}\left(\left.K\right|_{T} *_{\gamma} K^{\prime}\right) \subseteq K *_{\gamma} K^{\prime}$.

Proof. Let $K, K^{\prime} \in \mathcal{K}_{\mathcal{L}}$. Assume that $\gamma$ is a selection function for $K$ such that, for any $(\tau, \rho) \in \gamma\left(\mathbb{K}_{K^{\prime}, \subseteq}\right), \bigcap \gamma\left(\mathbb{T}_{K^{\prime}}\right) \subseteq(\tau, \emptyset)$ and $\gamma\left(\mathbb{P}_{\left.K\right|_{T} *_{\gamma} K^{\prime}}\right)=\left.\gamma\left(\mathbb{K}_{K^{\prime}, \subseteq}\right)\right|_{P}$. If $K^{\prime}$ is not satisfiable, then it follows directly from Definition 3 that $\left.K\right|_{P} *_{\gamma}\left(\left.K\right|_{T} *_{\gamma}\right.$

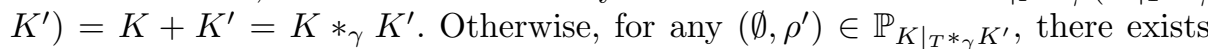

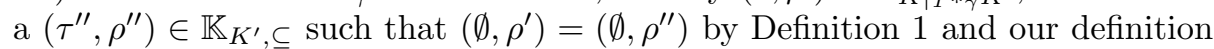
of $\mathbb{P}_{K^{\prime}}$ and $\left(\left.K\right|_{T} *_{\gamma} K^{\prime}\right) \backslash K^{\prime}=\bigcap \gamma\left(\mathbb{T}_{K^{\prime}}\right) \subseteq\left(\tau^{\prime \prime}, \emptyset\right)$ by Definitions 1 and 3 . Due to the assumption, it then follows that $\left.\bigcap \gamma\left(\mathbb{K}_{K^{\prime}, \subseteq}\right)\right|_{P}=\bigcap \gamma\left(\mathbb{P}_{\left.K\right|_{T^{*} \gamma} K^{\prime}}\right)$ and $\left.\bigcap \gamma\left(\mathbb{T}_{K^{\prime}}\right) \subseteq \bigcap \gamma\left(\mathbb{K}_{K^{\prime}, \subseteq}\right)\right|_{T}$. This implies $\left.K\right|_{P} *_{\gamma}\left(\left.K\right|_{T} *_{\gamma} K^{\prime}\right) \subseteq K *_{\gamma} K^{\prime}$ by Definition 3. 
Proposition 5. Let $K, K^{\prime} \in \mathcal{K}_{\mathcal{L}}$ There exists a selection function $\gamma$ for $K$ such that $\left.K\right|_{T} *_{\gamma}\left(\left.K\right|_{P} *_{\gamma} K^{\prime}\right) \subseteq K *_{\gamma} K^{\prime}$.

Proof. Let $K, K^{\prime} \in \mathcal{K}_{\mathcal{L}}$. Assume that $\gamma$ is a selection function for $K$ such that, for any $(\tau, \rho) \in \gamma\left(\mathbb{K}_{K^{\prime}, \subseteq}\right), \bigcap \gamma\left(\mathbb{P}_{K^{\prime}}\right) \subseteq(\emptyset, \rho)$ and $\gamma\left(\mathbb{T}_{\left.K\right|_{P} *_{\gamma} K^{\prime}}\right)=\left.\gamma\left(\mathbb{K}_{K^{\prime}, \subseteq}\right)\right|_{T}$. The proof then follows symmetrically to the proof of Proposition 4.

\subsection{Prioritised Revision}

While protected revision allows us to emphasise entire components of a HKB during a revision, its flexibility is somewhat limited. Specifically, in Example 3 above we saw that the theory component of $K$ was fully preserved due to the structure of the new information $K^{\prime}$. Intuitively, the revision outcome states that the unisex name Chris was incorrectly identified or entered as female. Yet, what if Chris is actually female and she married Kate after the law changed to allow same-sex marriage? In that case, we need to have a more fine-grained method than protected revision to allow the theory component to be changed. We therefore define prioritised revision now, based on an idea by Nebel [17].

Definition 5 (Priority Level). Let $K \in \mathcal{K}_{\mathcal{L}}$ and $\leq$ be a total preorder on $K_{T} \cup K_{P} . A$ priority level of $K$ is

$$
\lambda=\left\{(\tau, \rho) \subseteq K \mid e_{1} \leq e_{2} \text { and } e_{2} \leq e_{1} \text { for all } e_{1}, e_{2} \in \tau \cup \rho\right\} .
$$

We call $\leq$ defined as above a prioritisation of $K$. Furthermore, for all $e_{1} \in \lambda_{1}$ and $e_{2} \in \lambda_{2}$, we write $\lambda_{1} \leq \lambda_{2}$ iff $e_{1} \leq e_{2}$, and $\lambda_{1}<\lambda_{2}$ iff $e_{1} \leq e_{2}$ and $e_{2} \not \leq e_{1}$. A prioritisation partitions $K$ into a hierarchical sequence of levels and places each formula or rule of $K$ in a particular level. We modify our definition of compatible sets to include priority levels.

Definition 6 (Prioritised Compatible Set). Let $K, K^{\prime} \in \mathcal{K}_{\mathcal{L}}$ and $\leq$ be a prioritisation of $K$. The set of prioritised compatible sets of $K$ regarding $K^{\prime}$ is

$$
\mathbb{K}_{K^{\prime}, \leq}=\left\{(\tau, \rho) \mid(\tau, \rho)=\bigcup_{\lambda \subseteq K}\left(\tau_{\lambda}, \rho_{\lambda}\right)\right\},
$$

such that for all $\lambda \subseteq K$ :

a) $\left(\tau_{\lambda}, \rho_{\lambda}\right) \subseteq \lambda$,

b) $Q H T\left(\bigcup_{\lambda<\lambda^{\prime}}\left(\tau_{\lambda^{\prime}}, \rho_{\lambda^{\prime}}\right)\right) \cap Q H T\left(K^{\prime}\right) \neq \emptyset$, and

c) for all $\left(\tau_{\lambda}^{\prime}, \rho_{\lambda}^{\prime}\right) \subseteq \lambda,\left(\tau_{\lambda}, \rho_{\lambda}\right) \subset\left(\tau_{\lambda}^{\prime}, \rho_{\lambda}^{\prime}\right)$ implies $Q H T\left(\bigcup_{\lambda<\lambda^{\prime}}\left(\tau_{\lambda^{\prime}}, \rho_{\lambda^{\prime}}\right)\right) \cap$ $Q H T\left(\left(\tau_{\lambda}^{\prime}, \rho_{\lambda}^{\prime}\right)\right) \cap Q H T\left(K^{\prime}\right)=\emptyset$.

Each prioritised compatible set is composed of a maximal subset of the highest level that is compatible with $K^{\prime}$, a maximal subset of the next highest level that is compatible with $K^{\prime}$ and the subsets formed previously in higher levels, and so on. With these concepts established, we can now define a prioritised revision operator as follows. 
Definition 7 (Prioritised Revision). Let $K \in \mathcal{K}_{\mathcal{L}}$ and $\leq$ be a prioritisation of $K$. A prioritised revision operator $* \leq$ for $K$ is defined such that for any $K^{\prime} \in \mathcal{K}_{\mathcal{L}}:$

$$
K * \leq K^{\prime}= \begin{cases}K+K^{\prime} & \text { if } K^{\prime} \text { is not satisfiable }, \\ \bigcap \mathbb{K}_{K^{\prime}, \leq}+K^{\prime} & \text { otherwise. }\end{cases}
$$

Whenever $K^{\prime}$ is satisfiable, our prioritised revision operator $* \leq$ returns those elements of $K$ that are common to all prioritised compatible sets together with $K^{\prime}$. The following example illustrates the operation of prioritised revision and shows that it can indeed return the desired result with respect to our running example, that is, appropriately represent a change in marriage legislation.

Example 4. Consider again $K$ from Example 1 and $K^{\prime}$ from Example 3. Let $\leq$ be a prioritisation of $K$ such that assertion of the user's gender is given higher priority than prohibition of same-sex marriage. Formally, let $\lambda_{1}=(\{\forall x, y$ : $\operatorname{married}(x, y) \wedge$ female $(x) \rightarrow$ male $(y)\}, \emptyset), \lambda_{2}=(\emptyset,\{$ female $($ Chris $)\}),. \lambda_{3}=$ $K \backslash\left(\lambda_{1} \cup \lambda_{2}\right)$, and $\lambda_{1}<\lambda_{2}<\lambda_{3}$. It then follows that $\mathbb{K}_{K^{\prime}, \leq}=\left\{\lambda_{2} \cup \lambda_{3}\right\}$ and thus $K * \leq K^{\prime}=\lambda_{2} \cup \lambda_{3} \cup K^{\prime}$.

Comparing our prioritised revision operator to our partial meet revision operator, we find that they coincide, as stated in Propositions 6 and 7.

Proposition 6. Let $K, K^{\prime} \in \mathcal{K}_{\mathcal{L}}$. For any selection function $\gamma$ for $K$, there exists a prioritisation $\leq$ of $K$ such that $K *_{\gamma} K^{\prime}=K *_{\leq} K^{\prime}$.

Proof. Let $K, K^{\prime} \in \mathcal{K}_{\mathcal{L}}$ and $\gamma$ be a selection function for $K$ that determines the outcome of $K *_{\gamma} K^{\prime}$. By $S=\bigcap \gamma\left(\mathbb{K}_{K^{\prime}, \subseteq}\right)$ we denote the part of $K$ that is preserved in the partial meet revision and by $S^{\prime}=K \backslash S$ the part of $K$ that is discarded. We can then create a prioritisation $\leq$ of $K$ as follows. In the limiting case that $S=\emptyset$, let $\leq$ consist of one level $\lambda_{1}=S^{\prime}$ only. From Definitions 1 and 6 it then follows that $\mathbb{K}_{K^{\prime}, \subseteq}=\mathbb{K}_{K^{\prime}, \leq}$. Since $\gamma\left(\mathbb{K}_{K^{\prime}, \subseteq}\right) \subseteq \mathbb{K}_{K^{\prime}, \subseteq}$ by Definition 2 and $\bigcap \gamma\left(\mathbb{K}_{K^{\prime}, \subseteq}\right)=\emptyset$ by the assumption, we obtain $\bigcap \mathbb{K}_{K^{\prime}, \leq}=\emptyset$ and consequently $K *_{\gamma} K^{\prime}=K * \leq K^{\prime}$. Otherwise, let $\leq$ consist of exactly two levels $\lambda_{1}$ and $\lambda_{2}$ such that $\lambda_{1}=S^{\prime}, \lambda_{2}=S$, and $\lambda_{1}<\lambda_{2}$. We now show that $\bigcap \mathbb{K}_{K^{\prime}, \leq}=S$. For any $\kappa \in \mathbb{K}_{K^{\prime}, \leq}$, it follows from Definition 6 and our choice of $\leq$ that $S \subseteq \kappa$, which implies $S \subseteq \bigcap \mathbb{K}_{K^{\prime}, \leq}$ by Definition 7 . Assume that there exists a $\kappa^{\prime} \subseteq S^{\prime}$ such that $\kappa^{\prime} \subseteq \bigcap \mathbb{K}_{K^{\prime}, \leq}$. Then for each compatible set $\kappa^{\prime \prime} \in \mathbb{K}_{K^{\prime}, \subseteq}$ with $S \subseteq \kappa^{\prime \prime}$ it would hold that $\kappa^{\prime} \subseteq \kappa^{\prime \prime}$ because $\kappa^{\prime \prime}$ is maximal by Definition 1 . Yet, this would imply $\kappa^{\prime} \subseteq \bigcap \gamma\left(\mathbb{K}_{K^{\prime}, \subseteq}\right)$ and thus $\kappa^{\prime} \subseteq S$, a contradiction.

Proposition 7. Let $K, K^{\prime} \in \mathcal{K}_{\mathcal{L}}$. For any prioritisation $\leq$ of $K$, there exists a selection function $\gamma$ for $K$ such that $K * \leq K^{\prime}=K *_{\gamma} K^{\prime}$.

Proof. Let $K, K^{\prime} \in \mathcal{K}_{\mathcal{L}}$ and $\leq$ be a prioritisation of $K$ that determines the outcome of $K *_{\gamma} K^{\prime}$. In the trivial case that $K^{\prime}$ is not satisfiable, we have $K * \leq$ $K^{\prime}=K+K^{\prime}=K *_{\gamma} K^{\prime}$. Let $K^{\prime}$ be satisfiable in the following. We first show 
that for any prioritised compatible set $\kappa_{1} \in \mathbb{K}_{K^{\prime}, \leq}$, there exists a compatible set $\kappa_{2} \in \mathbb{K}_{K^{\prime}, \subseteq}$ such that $\kappa_{1}=\kappa_{2}$. It follows directly from Definitions 1 and 6 that $\kappa_{1} \subseteq \kappa_{2}$. Assume that $\kappa_{1} \subset \kappa_{2}$. From Definition 6 it would then follow that $Q H T\left(\kappa_{1}\right) \cap Q H T\left(\kappa_{2} \backslash \kappa_{1}\right) \cap Q H T\left(K^{\prime}\right)=\emptyset$. Yet, this is a contradiction since $Q H T\left(\kappa_{2}\right) \cap Q H T\left(K^{\prime}\right) \neq \emptyset$ by Definition 1 and $\kappa_{1}$ is required to be maximal by Definition 6 . Thus, for any $\kappa_{1} \in \mathbb{K}_{K^{\prime}, \leq}$ it holds that $\kappa_{1} \in \mathbb{K}_{K^{\prime}, \subset}$, that is, $\mathbb{K}_{K^{\prime}, \leq \subseteq} \subseteq \mathbb{K}_{K^{\prime}, \subseteq}$. We can now set $\gamma$ as a selection function for $K$ such that $\gamma\left(\mathbb{K}_{K^{\prime}, \subseteq}\right)=\mathbb{K}_{K^{\prime}, \leq}$ and consequently obtain $K *_{\leq} K^{\prime}=K *_{\gamma} K^{\prime}$.

Since prioritised revision and partial meet revision coincide, it also holds that prioritised revision generalises protected revision and further that prioritised revision is exactly characterised by the postulates $(* 1)-(* 5)$.

Corollary 1. Let $K, K^{\prime} \in \mathcal{K}_{\mathcal{L}}$. For any selection function $\gamma$ for $K$, there exists a prioritisation $\leq$ of $K$ such that $K *_{\gamma}, \subseteq_{\circ} K^{\prime}=K *_{\leq} K^{\prime}$.

Proof. Follows directly from Propositions 1 and 6.

Theorem 3. Let $K \in \mathcal{K}_{\mathcal{L}}$. An operator $* \leq$ is a prioritised revision operator for $K$ determined by a prioritisation $\leq$ of $\bar{K}$ iff $* \leq$ satisfies $(* 1)-(* 5)$.

Proof. Follows directly from Theorem 1 and Propositions 6 and 7.

\section{Related Work}

Closest related work concerns itself with belief update, a relative of belief revision, in hybrid MKNF knowledge bases [21], which combine first-order theories and logic programs in a different semantic composition to the one considered here. That approach takes the combination of an initial HKB and an updating HKB and splits it into hierarchical levels, similar to the process of logic program stratification, such that updating information is given priority. It then applies given ontology update and rule update semantics to the respective components to evaluate the models of the updated HKB. In contrast to our prioritised revision, the update method does not allow arbitrary combinations of formulas and rules on the same level. In addition, since the update operation preserves all existing information and relies on the chosen semantics to determine a satisfiable outcome, the initial HKB grows with each operation and may contain duplicate elements to express information that was first asserted, then contradicted, and later asserted again.

Multi-context systems allow reasoning over two or more knowledge bases, specified in some monotonic or nonmonotonic logic, by connecting them via nonmonotonic bridge rules. An adaptation of Dalal's [5] and Satoh's [20] classic belief revision operators to multi-context systems [22] computes the outcome of revising one multi-context system by another as the set of those models of the revising multi-context system that are closest to the ones of the initial multicontext system, where closeness is determined by Dalal's or Satoh's notion of 
distance. The adaptation to multi-context systems exhibits the same properties as the classic construction with respect to the belief revision postulates.

To assist with the task of rule authoring in HKBs, inductive logic programming can be used to learn new rules from the existing rules and formulas in a HKB [15]. As any induced rule is consistent with existing knowledge and no existing knowledge is removed, this process can be compared to screened revision [16] where the entire existing HKB is protected.

\section{Conclusion}

In this study, we proposed three operators to revise hybrid knowledge bases, namely, a general partial meet revision operator that does not assume any ordering of the elements of a hybrid knowledge base, a protected revision operator that emphasises the theory or program component of a hybrid knowledge base depending on the structure of information in the revising hybrid knowledge base, and lastly a prioritised revision operator that computes the outcome based on a pre-defined priority ordering among the elements in a hybrid knowledge base. We demonstrated the interdefinability between our operators, provided representation theorems for partial meet revision and prioritised revision with respect to the belief revision postulates, and showed that partial meet revision as well as prioritised revision are generalisations of protected revision.

It is straightforward to define corresponding partial meet, protected, and prioritised contraction operators, by using the complement of $Q H T\left(K^{\prime}\right)$ in the definitions of compatible sets. Properties and representation theorems analogous to revision can then be obtained, after translating the classic contraction postulates in a similar manner as the ones for revision.

While our operators return a revised hybrid knowledge base, they do not specify how a selection function or prioritisation changes during the operation. In future work, we aim to investigate such an extension to our approach.

\section{References}

1. Alchourrón, C.E., Gärdenfors, P., Makinson, D.: On the logic of theory change: Partial meet contraction and revision functions. Journal of Symbolic Logic 50(2), 510-530 (1985)

2. Binnewies, S., Zhuang, Z., Wang, K.: Partial meet revision and contraction in logic programs. In: Proceedings of the Twenty-Ninth AAAI Conference on Artificial Intelligence, AAAI 2015. pp. 1439-1445. AAAI Press, Palo Alto (2015)

3. de Bruijn, J., Pearce, D., Polleres, A., Valverde, A.: A semantical framework for hybrid knowledge bases. Knowledge and Information Systems 25(1), 81-104 (2010)

4. Colmerauer, A., Roussel, P.: The birth of prolog. In: Bergin, Jr., T.J., Gibson, Jr., R.G. (eds.) History of Programming languages-II, pp. 331-367. ACM, New York, NY, USA (1996)

5. Dalal, M.: Investigations into a theory of knowledge base revision: Preliminary report. In: Proceedings of the Seventh National Conference on Artificial Intelligence. pp. 475-479 (1988) 
6. Delgrande, J.P., Schaub, T., Tompits, H., Woltran, S.: A model-theoretic approach to belief change in answer set programming. ACM Transactions on Computational Logic 14(2), 14:1-14:46 (2013)

7. Drabent, W., Eiter, T., Ianni, G., Krennwallner, T., Lukasiewicz, T., Małuszyński, J.: Hybrid reasoning with rules and ontologies. In: Bry, F., Małuszyński, J. (eds.) Semantic Techniques for the Web: The REWERSE Perspective, LNCS, vol. 5500, pp. 1-49. Springer Berlin Heidelberg (2009)

8. Fuhrmann, A.: Theory contraction through base contraction. Journal of Philosophical Logic 20(2), 175-203 (1991)

9. Guarino, N., Oberle, D., Staab, S.: What is an ontology? In: Staab, S., Studer, R. (eds.) Handbook on Ontologies, pp. 1-17. International Handbooks on Information Systems, Springer Berlin Heidelberg (2009)

10. Hansson, S.O.: New operators for theory change. Theoria 55(2), 114-132 (1989)

11. Hansson, S.O.: Reversing the levi identity. Journal of Philosophical Logic 22(6), 637-669 (1993)

12. Hitzler, P., Parsia, B.: Ontologies and rules. In: Staab, S., Studer, R. (eds.) Handbook on Ontologies, pp. 111-132. International Handbooks on Information Systems, Springer Berlin Heidelberg (2009)

13. Kowalski, R.: Predicate logic as a programming language. In: Proceedings of the IFIP Congress. pp. 569-574 (1974)

14. Krisnadhi, A., Maier, F., Hitzler, P.: OWL and rules. In: Polleres, A., d'Amato, C., Arenas, M., Handschuh, S., Kroner, P., Ossowski, S., Patel-Schneider, P. (eds.) Reasoning Web. Semantic Technologies for the Web of Data, LNCS, vol. 6848, pp. 382-415. Springer Berlin Heidelberg (2011)

15. Lisi, F.A.: Learning onto-relational rules with inductive logic programming. In: Lehmann, J., Völker, J. (eds.) Perspectives on Ontology Learning, pp. 93-111. Studies on the Semantic Web, IOS Press Amsterdam (2014)

16. Makinson, D.: Screened revision. Theoria 63(1-2), 14-23 (1997)

17. Nebel, B.: Belief revision and default reasoning: Syntax-based approaches. In: Proceedings of the 2nd International Conference on Principles of Knowledge Representation and Reasoning (KR'91). pp. 417-428. Morgan Kaufmann, San Francisco (1991)

18. Pearce, D., Valverde, A.: Quantified equilibrium logic and foundations for answer set programs. In: Garcia de la Banda, M., Pontelli, E. (eds.) Logic Programming. ICLP 2008., LNCS, vol. 5366, pp. 546-560. Springer Berlin Heidelberg (2008)

19. Rott, H.: Modellings for belief change: Base contraction, multiple contraction, and epistemic entrenchment (preliminary report). In: Pearce, D., Wagner, G. (eds.) Logics in Artificial Intelligence, LNCS, vol. 633, pp. 139-153. Springer Berlin Heidelberg (1992)

20. Satoh, K.: Nonmonotonic reasoning by minimal belief revision. In: Proceedings of the International Conference on Fifth Generation Computer Systems. pp. 455-462 (1988)

21. Slota, M., Leite, J., Swift, T.: On updates of hybrid knowledge bases composed of ontologies and rules. Artificial Intelligence 229, 33-104 (2015)

22. Wang, Y., Zhuang, Z., Wang, K.: Belief change in nonmonotonic multi-context systems. In: Cabalar, P., Son, T.C. (eds.) LPNMR 2013. LNCS, vol. 8148, pp. 543-555. Springer Berlin Heidelberg (2013)

23. Wassermann, R.: On AGM for non-classical logics. Journal of Philosophical Logic 40(2), 271-294 (2011)

24. Zhuang, Z., Wang, Z., Wang, K., Qi, G.: DL-Lite contraction and revision. Journal of Artificial Intelligence Research 56(1), 329-378 (2016) 